\title{
ON $F_{0}$-NORMALITY AND ADDITIVE AUTOMORPHIC FUNCTIONS OF THE FIRST KIND
}

\author{
RAUNO AULASKARI
}

\section{Introduction and definitions}

1.1. In [1] we defined the notion of an additive automorphic function of the first kind. There we decomposed the class of additive automorphic functions into two subclasses, the classes of additive automorphic functions of the first kind and those of the second kind. For normal meromorphic functions the corresponding notion was given by Noshiro in [10] (cf. also [12]). We remarked in [1] that the definition given by Noshiro is not suitable for normal additive automorphic functions provided some period is non-zero. Here, in addition to the above subclasses, we shall define $F_{0}$-normality for additive automorphic functions, which is a weaker restriction than the usual normality. In Chapter 2 of this paper we proceed with the study of additive automorphic functions of the first kind. Further we give certain regularity conditions implying an additive automorphic function to be of the second kind. Integrable automorphic forms are considered in Chapter 3. In the final chapters we consider $F_{0}$-normal additive automorphic functions and their properties.

Let $D$ be the unit disk $\{z|| z \mid<1\}$ and $\Omega$ the totality of all Moebius transformations of $D$ onto itself. A function $f$, meromorphic in $D$, is called normal if the family $\{f \circ T \mid T \in \Omega\}$ is a normal family in the sense of Montel in $D$ (with respect to the spherical metric). An analytic function $f$ is called a Bloch function if

$$
\sup _{z \in D}\left(1-|z|^{2}\right)\left|f^{\prime}(z)\right|<\infty .
$$

We denote the hyperbolic distance by $d\left(z_{1}, z_{2}\right)\left(z_{1}, z_{2} \in D\right)$ and the spherical distance by $d^{*}\left(w_{1}, w_{2}\right)\left(w_{1}, w_{2} \in \hat{\boldsymbol{C}}\right)$. The hyperbolic disk $\left\{z \mid d\left(z, z_{0}\right)<r\right\}$ is denoted by $U\left(z_{0}, r\right)$. The euclidean disk is denoted by $D\left(z_{0}, s\right)$. A sequence of points $\left(z_{n}\right)$ in $D$ tending to $\partial D$ is said to be a sequence of $P$-points for a meromorphic function $f$ if for each $r>0$, and every infinite subsequence of points $\left(z_{k}\right)$ of $\left(z_{n}\right), f$ assumes every value, except perhaps two, infinitely often in the set $\bigcup_{k=1}^{\infty} U\left(z_{k}, r\right)$.

Let $\Gamma$ be a Fuchsian group, that is, a discontinuous subgroup of $\Omega$. The points $z, z^{\prime} \in \bar{D}$ are called $\Gamma$-equivalent if there exists a mapping $T \in \Gamma$ such that $z^{\prime}=T(z)$. A domain $F \subset D$ is called a fundamental domain of $\Gamma$ if it does not contain two 
$\Gamma$-equivalent points and if every point in $D$ is $\Gamma$-equivalent to some point in $\bar{F}$. We fix a fundamental domain $F_{0}$ to be some normal polygon containing the origin [7, IV. 7].

1.2. Definition. The fundamental domain $F_{0}$ is called thick if there exist positive constants $r, r^{\prime}$ such that for each sequence of points $\left(z_{n}\right) \subset F_{0}$ there is a sequence of points $\left(z_{n}^{\prime}\right)$ for which $d\left(z_{n}, z_{n}^{\prime}\right) \leqq r$ and $\mathrm{U}\left(z_{n}^{\prime}, r^{\prime}\right) \subset F_{0}$ for each $n=1,2, \ldots$.

1.3. Remark. Suppose that the fundamental domain $F_{0}$ is thick. Let $s>0$ be fixed. Then by the thickness of fundamental domains $T\left(F_{0}\right), T \in \Gamma$, there is a $n_{0}(s) \in N$ such that $U(z, s)$ has common points with at most $n_{0}(s)$ sets $T\left(\bar{F}_{0}\right), T \in \Gamma$.

A function $W^{\prime}$ is an automorphic form with respect to $\Gamma$, provided $W^{\prime}$ is meromorphic in $D$ and satisfies

$$
W^{\prime}(T(z))=\frac{1}{T^{\prime}(z)} W^{\prime}(z) \quad \text { for all } \quad z \in D, T \in \Gamma .
$$

If all residues of $W^{\prime}$ vanish, then $W^{\prime}$ has

$$
W(z)=\int_{0}^{z} W^{\prime}(t) d t, \quad z \in D,
$$

as an integral function and it satisfies

$$
W(T(z))=\int_{0}^{T(z)} W^{\prime}(t) d t=W(z)+A_{T}, \quad T \in \Gamma,
$$

where $A_{T}$ is called the period of $W$ with respect to $T$. The integral function $W$ will be called an additive automorphic function with respect to $\Gamma$.

1.4. Definition. Let $W$ be an additive automorphic function with respect to $\Gamma$. The function $W$ is said to be of the second kind if there exists a sequence of points $\left(z_{n}\right)$ in the closure $\bar{F}_{0}$ such that the sequence of functions

$$
g_{n}(\zeta)=W\left(\frac{\zeta+z_{n}}{1+\bar{z}_{n} \zeta}\right), \quad n=1,2, \ldots,
$$

tends uniformly to a constant limit in some neighbourhood of $\zeta=0$. An additive automorphic function $W$ in $D$ is said to be of the first kind if it is not of the second kind.

1.5. Definition. An additive automorphic function $W$ is called $F_{0}$-normal if for each sequence of points $\left(z_{n}\right)$ in the closure $\bar{F}_{0}$ the sequence of functions

forms a normal family in $D$.

$$
g_{n}(\zeta)=W\left(\frac{\zeta+z_{n}}{1+\bar{z}_{n} \zeta}\right), \quad n=1,2, \ldots,
$$


An automorphic form $W^{\prime}$ is called integrable if

$$
\iint_{F_{0}} \frac{1}{1-|z|^{2}}\left|W^{\prime}(z)\right| d \sigma_{z}<\infty,
$$

where $d \sigma_{z}$ is the euclidean area element.

\section{On additive automorphic functions of the first kind}

In the first theorem of this chapter we deal with $P$-sequences for additive automorphic functions of the first kind.

2.1. Theorem. Let $W$ be an analytic additive automorphic function of the first kind. If $W$ is not a Bloch function, then each fundamental domain $F_{n}=T_{n}\left(F_{0}\right), T_{n} \in \Gamma$, possesses a sequence of P-points for $W$.

Proof. Since $W$ is an additive automorphic function, it is sufficient to prove that there is a sequence of $P$-points for $W$ in $F_{0}$. By the assumption there is a sequence of points $\left(z_{n}\right) \subset F_{0}$ such that $\left(1-\left|z_{n}\right|^{2}\right)\left|W^{\prime}\left(z_{n}\right)\right| \rightarrow \infty$ for $n \rightarrow \infty$. We assert that $\left(z_{n}\right)$ is a sequence of $P$-points for $W$. Suppose, on the contrary, that $\left(z_{n}\right)$ is not a sequence of $P$-points for $W$. We may then assume that there exists a finite positive number $r$ such that $W$ omits two finite values in $\bigcup_{k=1}^{\infty} U\left(z_{k}, r\right)$ where $\left(z_{k}\right)$ is a subsequence of $\left(z_{n}\right)$. Hence the sequence of functions

$$
g_{k}(\zeta)=W\left(\frac{\zeta+z_{k}}{1+\bar{z}_{k} \zeta}\right), \quad k=1,2, \ldots,
$$

omits two finite values in $U(0, r)$ and thus forms there a normal family. This implies that the sequence of functions (2.1) possesses a subsequence $\left(g_{h}\right)$ such that $\lim _{h \rightarrow \infty} g_{h}(\zeta)=g(\zeta)$ uniformly on every compact part of $U(0, r)$. Since $W$ is of the first kind, $g$ is an analytic function in $U(0, r)$ (and not the constant $\infty$ ). On the other hand,

$$
\begin{gathered}
\left|g^{\prime}(0)\right|=\lim _{h \rightarrow \infty}\left|g_{h}^{\prime}(0)\right| \\
=\lim _{h \rightarrow \infty}\left(1-\left|z_{h}\right|^{2}\right)\left|W^{\prime}\left(z_{h}\right)\right|=\infty,
\end{gathered}
$$

which is a contradiction. Thus the theorem is proved.

We give now a sufficient and necessary condition for an additive automorphic function to be of the first kind (cf. [10, Theorem 6], [12, Theorem 1]).

2.2. Theorem. Let $W$ be an additive automorphic function with respect to $\Gamma$. Then $W$ is of the first kind if and only if

$$
\liminf _{a \in \bar{F}_{0}} \iint_{U(a, r)}\left(\frac{\left|W^{\prime}(z)\right|}{1+|W(z)|^{2}}\right)^{2} d \sigma_{z}>0
$$

for any positive number $r$. 
Proof. Let $W$ be of the first kind. Suppose, on the contrary, that

$$
\lim _{n \rightarrow \infty} \iint_{U\left(z_{n}, r\right)}\left(\frac{\left|W^{\prime}(z)\right|}{1+\left|W^{\prime}(z)\right|^{2}}\right)^{2} d \sigma_{z}=0
$$

for some sequence of points $\left(z_{n}\right) \subset \bar{F}_{0}$ and some $r>0$. Without loss of generality, it may be supposed that

$$
\sum_{n=1}^{\infty} \iint_{U\left(z_{n}, r\right)}\left(\frac{\left|W^{\prime}(z)\right|}{1+|W(z)|^{2}}\right)^{2} d \sigma_{z}<\pi
$$

We consider the functions

$$
g_{n}(\zeta)=W\left(\frac{\zeta+z_{n}}{1+\bar{z}_{n} \zeta}\right), \quad n=1,2, \ldots
$$

Now $\iint_{U(0, r)}\left(\left|g_{n}^{\prime}(\zeta)\right| /\left(1+\left|g_{n}(\zeta)\right|^{2}\right)\right)^{2} d \sigma_{\zeta}$ is equal to the spherical area of the surface onto which $g_{n}$ maps the disk $U(0, r)$. Hence by $(2.5)$ and the equation

$$
\iint_{U(0, r)}\left(\frac{\left|g_{n}^{\prime}(\zeta)\right|}{1+\left|g_{n}(\zeta)\right|^{2}}\right)^{2} d \sigma_{\zeta}=\iint_{U\left(z_{n}, r\right)}\left(\frac{\left|W^{\prime}(z)\right|}{1+|W(z)|^{2}}\right)^{2} d \sigma_{z}
$$

the family $\left\{g_{n}\right\}$ omits three values in $U(0, r)$ and thus forms there a normal family. Let $\left(g_{k}\right)$ be a subsequence of $\left(g_{n}\right)$ converging uniformly to a meromorphic function $g$ on every compact part of $U(0, r)$. Since $W$ is of the first kind, $g$ is non-constant. By the uniform convergence

$$
\begin{aligned}
& \lim _{k \rightarrow \infty} \iint_{U\left(0, r^{\prime}\right)}\left(\frac{\left|g_{k}^{\prime}(\zeta)\right|}{1+\left|g_{k}(\zeta)\right|^{2}}\right)^{2} d \sigma_{\zeta} \\
= & \iint_{U\left(0, r^{\prime}\right)}\left(\frac{\left|g^{\prime}(\zeta)\right|}{1+|g(\zeta)|^{2}}\right)^{2} d \sigma_{\zeta}>0
\end{aligned}
$$

with $0<r^{\prime}<r$. This contradicts (2.4).

If $W$ is of the second kind, there is a sequence of points $\left(z_{n}\right) \subset \bar{F}_{0}$ such that the sequence of functions $\left(g_{n}(\zeta)\right)=\left(W\left(\left(\zeta+z_{n}\right) /\left(1+\bar{z}_{n} \zeta\right)\right)\right)$ converges uniformly to a constant limit in the hyperbolic disk $U(0, r)$ for some $r>0$. This implies that the sequence of real-valued functions $\left(\left|g_{n}^{\prime}(\zeta)\right| /\left(1+\left|g_{n}(\zeta)\right|^{2}\right)\right)$ tends to zero there, too. Hence, for all $0<r^{\prime}<r$,

On the other hand, we have

$$
\lim _{n \rightarrow \infty} \iint_{U\left(0, r^{\prime}\right)}\left(\frac{\left|g_{n}^{\prime}(\zeta)\right|}{1+\left|g_{n}(\zeta)\right|^{2}}\right)^{2} d \sigma_{\zeta}=0 .
$$

$$
\begin{aligned}
& \lim _{n \rightarrow \infty} \iint_{U\left(z_{n}, r^{\prime}\right)}\left(\frac{\left|W^{\prime}(z)\right|}{1+|W(z)|^{2}}\right)^{2} d \sigma_{z} \\
= & \lim _{n \rightarrow \infty} \iiint_{U\left(0, r^{\prime}\right)}\left(\frac{\left|g_{n}^{\prime}(\zeta)\right|}{1+\left|g_{n}(\zeta)\right|^{2}}\right)^{2} d \sigma_{\zeta},
\end{aligned}
$$

and thus the theorem is proved. 
2.3. Corollary. Let $W$ be an additive automorphic function with respect to $\Gamma$ and let the fundamental domain $F_{0}$ be non-compact and thick. If $\iint_{F_{0}}\left(\left|W^{\prime}(z)\right| /\left(1+|W(z)|^{2}\right)\right)^{2} d \sigma_{z}<\infty$, then $W$ is of the second kind in $D$.

Proof. Suppose, on the contrary, that $W$ is of the first kind in $D$. By the thickness and non-compactness of the fundamental domain $F_{0}$ we are able to choose the sequence of hyperbolic disks $\left(U\left(z_{n}, r\right)\right) \subset F_{0}$ such that $\left|z_{n}\right| \rightarrow 1$ for $n \rightarrow \infty$. Further, by the boundedness of the integral $\iint_{F_{0}}\left(\left|W^{\prime}(z)\right| /\left(1+|W(z)|^{2}\right)\right)^{2} d \sigma_{z}$ we have $\iint_{U\left(z_{n}, r\right)}\left(\left|W^{\prime}(z)\right| /\left(1+|W(z)|^{2}\right)\right)^{2} d \sigma_{z} \rightarrow 0$ for $n \rightarrow \infty$. This contradicts 2.2, and thus the corollary is proved.

2.4. Remark. For proving 2.3 it is sufficient, instead of the thickness of $F_{0}$, to find a sequence of hyperbolic disks $\left(U\left(z_{n}, r\right)\right) \subset F_{0}$ such that $\left|z_{n}\right| \rightarrow 1$ for $n \rightarrow \infty$.

2.5. Corollary. Let $W$ be an additive automorphic function of the first kind and let the fundamental domain $F_{0}$ be thick. If $\iint_{F_{0}}\left(\left|W^{\prime}(z)\right| /\left(1+|W(z)|^{2}\right)\right)^{2} d \sigma_{z}<\infty$, then $\iint_{G_{R}}\left(\left|W^{\prime}(z)\right|^{\prime} /\left(1+|W(z)|^{2}\right)\right)^{2} d \sigma_{z}<\infty$ for each $G_{R}=\left\{z \mid d\left(z, F_{0}\right)<R\right\}$.

Proof. By 2.3 the fundamental domain $F_{0}$ is compact and hence $\bar{G}_{R}$ is compact, too. Thus the corollary is proved.

2.6. Corollary. Let $W$ be an additive automorphic function of the first kind. Then

$$
\liminf _{a \in D} \iint_{U(a, r)}\left|W^{\prime}(z)\right|^{2} d \sigma_{z}>0
$$

for any positive number $r$.

Proof. Let $\left(z_{n}\right) \subset D$ be any sequence of points and $r$ any positive number. Choose $T_{n} \in \Gamma$ such that $T_{n}\left(z_{n}\right)=z_{n}^{\prime} \in \bar{F}_{0}$ for each $n=1,2, \ldots$. Then

$$
\begin{gathered}
\lim \inf \iint_{U\left(z_{n}, r\right)}\left|W^{\prime}(z)\right|^{2} d \sigma_{z}=\lim \inf \iint_{U\left(z_{n}^{\prime}, r\right)}\left|W^{\prime}(z)\right|^{2} d \sigma_{z} \\
\geqq \liminf \iint_{U\left(z_{n}^{\prime}, r\right)}\left(\frac{\left|W^{\prime}(z)\right|}{1+|W(z)|^{2}}\right)^{2} d \sigma_{z} \geqq m_{r}>0,
\end{gathered}
$$

where $m_{r}$ depends only on $r$ by 2.2. The corollary follows.

\section{On integrable automorphic forms}

In the first theorem of this chapter we consider an integrable automorphic form $W^{\prime}$ with respect to $\Gamma$ in the case the fundamental domain $F_{0}$ is thick. This corresponds to 3.7 Theorem in [4].

3.1. Theorem. Let $W^{\prime}$ be an integrable automorphic form with respect to $\Gamma$ and the fundamental domain $F_{0}$ thick. Then $W$ is a Bloch function in $D$. 
Proof. Suppose, on the contrary, that $W$ is not a Bloch function in $D$. Then there is a sequence of points $\left(z_{n}\right) \subset F_{0}$ such that $\left(1-\left|z_{n}\right|^{2}\right)\left|W^{\prime}\left(z_{n}\right)\right| \rightarrow \infty$ for $n \rightarrow \infty$. We consider the hyperbolic disks $U\left(z_{n}, r\right), n=1,2, \ldots$, for some $r>0$. There is a $k_{0} \in N$ such that

$$
D\left(z_{n},\left(1 / k_{0}\right)\left(1-\left|z_{n}\right|\right)\right)=D\left(z_{n}, s_{n}\right) \subset U\left(z_{n}, r\right)
$$

for each $n=1,2, \ldots$ By Cauchy's integral formula we have

$$
W^{\prime}\left(z_{n}\right)=\frac{1}{\pi s_{n}^{2}} \underset{D\left(z_{n}, s_{n}\right)}{\int} W^{\prime}(z) d \sigma_{z}
$$

Let $z \in D\left(z_{n}, s_{n}\right)$. Then $\left(1-\left|z_{n}\right|\right) /(1-|z|) \geqq\left(1-\left|z_{n}\right|\right) /\left(\left(k_{0}+1\right) / k_{0}\right)\left(1-\left|z_{n}\right|\right)=k_{0} /\left(k_{0}+1\right)$ and $\left(1-\left|z_{n}\right|\right)^{-1} \leqq\left(\left(k_{0}+1\right) / k_{0}\right)(1-|z|)^{-1}$ for each $z \in D\left(z_{n}, s_{n}\right)$. Therefore

$$
\begin{aligned}
& \left(1-\left|z_{n}\right|^{2}\right)\left|W^{\prime}\left(z_{n}\right)\right| \leqq 2\left(1-\left|z_{n}\right|\right)\left|W^{\prime}\left(z_{n}\right)\right| \\
& \leqq \frac{2 k_{0}^{2}}{\pi} \iint_{D\left(z_{n}, s_{n}\right)} \frac{1}{1-\left|z_{n}\right|}\left|W^{\prime}(z)\right| d \sigma_{z} \\
& \leqq \frac{4 k_{0}\left(k_{0}+1\right)}{\pi} \iiint_{D\left(z_{n}, s_{n}\right)} \frac{1}{1-|z|^{2}}\left|W^{\prime}(z)\right| d \sigma_{z} \\
& \leqq \frac{4 k_{0}\left(k_{0}+1\right)}{\pi} \iint_{U\left(z_{n}, r\right)} \frac{1}{1-|z|^{2}}\left|W^{\prime}(z)\right| d \sigma_{z}
\end{aligned}
$$

and hence $\iint_{U\left(z_{n}, r\right)}\left(1 /\left(1-|z|^{2}\right)\right)\left|W^{\prime}(z)\right| d \sigma_{z} \rightarrow \infty$ for $n \rightarrow \infty$. By the boundedness of the integral $\iint_{F_{0}}\left(1 /\left(1-|z|^{2}\right)\right)\left|W^{\prime}(z)\right| d \sigma_{z} \quad \Gamma$-equivalent points begin to appear in an increasing number in $U\left(z_{n}, r\right), n=1,2, \ldots$ This is contradictory to 1.3 , and thus the theorem is proved.

3.2. Remark. If the fundamental domain $F_{0}$ is thick, there is a lower bound greater than 2 for the traces of the hyperbolic transformations in $\Gamma$ [9, Theorem 2].

3.3. Theorem. Let $W^{\prime}$ be an integrable automorphic form with respect to $\Gamma$ and let $\left(z_{n}\right) \subset F_{0}$ be a sequence of points such that $\left(1-\left|z_{n}\right|^{2}\right)\left|W^{\prime}\left(z_{n}\right)\right| \rightarrow \infty$ for $n \rightarrow \infty$. If $U\left(z_{n}, r_{n}\right)$ is the largest hyperbolic disk with center at $z_{n}$ contained in $F_{0}$, then $\lim _{n \rightarrow \infty} r_{n}=0$.

Proof. Suppose, on the contrary, that there is a subsequence $\left(r_{k}\right)$ of $\left(r_{n}\right)$ such that $r_{k} \geqq r_{0}>0$ for each $k=1,2, \ldots$. Then there is a $k_{0} \in N$ such that

$$
D\left(z_{k},\left(1 / k_{0}\right)\left(1-\left|z_{k}\right|\right)\right)=D\left(z_{k}, s_{k}\right) \subset U\left(z_{k}, r_{0}\right),
$$


$k=1,2, \ldots$ By the consideration of 3.1 we obtain

$$
\begin{aligned}
& \left(1-\left|z_{k}\right|^{2}\right)\left|W^{\prime}\left(z_{k}\right)\right| \\
& \leqq \frac{4 k_{0}\left(k_{0}+1\right)}{\pi} \int_{D\left(z_{k}, s_{k}\right)} \frac{1}{1-|z|^{2}}\left|W^{\prime}(z)\right| d \sigma_{z} \\
& \leqq \frac{4 k_{0}\left(k_{0}+1\right)}{\pi} \iint_{F_{0}} \frac{1}{1-|z|^{2}}\left|W^{\prime}(z)\right| d \sigma_{z}<\infty
\end{aligned}
$$

for each $k=1,2, \ldots$. This is a contradiction, and thus the proof of the theorem is complete.

\section{Estimate for the spherical derivative}

In this chapter we shall see that the spherical derivative of a $F_{0}$-normal function in any hull of $F_{0}$ can be estimated from above in the same way as that of a normal function in $D$.

4.1. Theorem. Let $W$ be an additive automorphic function with respect to $\Gamma$. Then $W$ is a $F_{0}$-normal function if and only if there exists for every $R>0$ a finite $C_{R}>0$ such that

$$
\frac{\left|W^{\prime}(z)\right|}{1+|W(z)|^{2}} \leqq \frac{C_{R}}{1-|z|^{2}}
$$

for each $z \in G_{R}=\left\{z \mid d\left(z, F_{0}\right)<R\right\}$.

Proof. The condition is sufficient, for if $W$ is not a $F_{0}$-normal function, there is a sequence of points $\left(z_{n}^{\prime}\right) \subset \bar{F}_{0}$ such that the family $\left\{g_{n}(\zeta)\right\}=\left\{W\left(\left(\zeta+z_{n}^{\prime}\right) /\left(1+\bar{z}_{n}^{\prime} \zeta\right)\right)\right\}$ is not normal in $D$. Therefore there is a sequence of points $\left(\zeta_{k}\right) \subset U(0, R)$, for some $R>0$, such that

$$
\frac{\left|g_{k}^{\prime}\left(\zeta_{k}\right)\right|}{1+\left|g_{k}\left(\zeta_{k}\right)\right|^{2}} \rightarrow \infty
$$

as $k \rightarrow \infty$. Denote $z_{k}=\left(\zeta_{k}+z_{k}^{\prime}\right) /\left(1+\bar{z}_{k}^{\prime} \zeta_{k}\right)$. Then

$$
\begin{gathered}
\left(1-\left|z_{k}\right|^{2}\right) \frac{\left|W^{\prime}\left(z_{k}\right)\right|}{1+\left|W\left(z_{k}\right)\right|^{2}} \\
=\left(1-\delta\left(z_{k}^{\prime}, z_{k}\right)^{2}\right) \frac{\left|g_{k}^{\prime}\left(\zeta_{k}\right)\right|}{1+\left|g_{k}\left(\zeta_{k}\right)\right|^{2}},
\end{gathered}
$$

where $\delta\left(z_{k}^{\prime}, z_{k}\right)=\left|z_{k}^{\prime}-z_{k}\right| /\left|1-\bar{z}_{k}^{\prime} z_{k}\right|$. Now $\quad 1-\delta\left(z_{k}^{\prime}, z_{k}\right)^{2} \geqq r>0 \quad$ for $\quad d\left(z_{k}^{\prime}, z_{k}\right)=$ $d\left(0, \zeta_{k}\right)<R$. Consequently,

which is a contradiction.

$$
\lim _{k \rightarrow \infty}\left(1-\left|z_{k}\right|^{2}\right) \frac{\left|W^{\prime}\left(z_{k}\right)\right|}{1+\left|W\left(z_{k}\right)\right|^{2}}=\infty,
$$


The condition is also necessary. Indeed, suppose, on the contrary, that there exists a sequence of points $\left(z_{n}\right) \subset G_{R}$, for some $R>0$, such that

$$
\frac{\left|W^{\prime}\left(z_{n}\right)\right|}{1+\left|W\left(z_{n}\right)\right|^{2}}>\frac{n}{1-\left|z_{n}\right|^{2}}
$$

for each $n=1,2, \ldots$. Choose a sequence of points $\left(z_{n}^{\prime}\right) \subset \bar{F}_{0}$ such that $d\left(z_{n}, z_{n}^{\prime}\right)<R$ for each $n=1,2, \ldots$ By the assumption $\left\{g_{n}(\zeta)\right\}=\left\{W\left(\left(\zeta+z_{n}^{\prime}\right) /\left(1+\bar{z}_{n}^{\prime} \zeta\right)\right)\right\}$ is a normal family in $D$. Therefore the family of their spherical derivatives is bounded on every compact part of $D$ and thus $\left|g_{n}^{\prime}(\zeta)\right| /\left(1+\left|g_{n}(\zeta)\right|^{2}\right) \leqq M<\infty$ for each $\zeta \in U(0, R)$. Denote $\zeta_{n}=\left(z_{n}-z_{n}^{\prime}\right) /\left(1-\bar{z}_{n}^{\prime} z_{n}\right)$. Then $d\left(0, \zeta_{n}\right)=d\left(z_{n}^{\prime}, z_{n}\right)<R$ for each $n=1,2, \ldots$ and hence

It follows that

$$
\frac{\left|g_{n}^{\prime}\left(\zeta_{n}\right)\right|}{1+\left|g_{n}\left(\zeta_{n}\right)\right|^{2}}=\frac{\left|1-\bar{z}_{n}^{\prime} z_{n}\right|^{2}}{1-\left|z_{n}^{\prime}\right|^{2}} \cdot \frac{\left|W^{\prime}\left(z_{n}\right)\right|}{1+\left|W\left(z_{n}\right)\right|^{2}} \leqq M .
$$

$$
\begin{aligned}
& \frac{\left|W^{\prime}\left(z_{n}\right)\right|}{1+\left|W\left(z_{n}\right)\right|^{2}} \leqq \frac{M}{1-\left|z_{n}\right|^{2}} \cdot\left(1-\delta\left(z_{n}, z_{n}^{\prime}\right)^{2}\right) \\
& \leqq \frac{M}{1-\left|z_{n}\right|^{2}},
\end{aligned}
$$

where $\delta\left(z_{n}, z_{n}^{\prime}\right)=\left|z_{n}-z_{n}^{\prime}\right| /\left|1-\bar{z}_{n}^{\prime} z_{n}\right|$, for each $n=1,2, \ldots$ This contradicts (4.4), and thus the theorem is proved.

4.2. Corollary. $A F_{0}$-normal automorphic function $W$ is normal in $D$.

Proof. The assertion follows from the equation

$$
\sup _{z \in D}\left(1-|z|^{2}\right)\left|W^{\prime}(z)\right| /\left(1+|W(z)|^{2}\right)=\sup _{z \in F_{0}}\left(1-|z|^{2}\right)\left|W^{\prime}(z)\right| /\left(1+|W(z)|^{2}\right)
$$

by use of 4.1 .

After this, in both theorems of this chapter, we suppose that $\Gamma$ is a finitely generated Fuchsian group and the additive automorphic function $W$ has a meromorphic behaviour at parabolic vertices (this is equivalent to the assumption that $W$ has angular limits at parabolic vertices $[7, \mathrm{~V} 1 \mathrm{C}]$ ). We study first the connection between $F_{0}$-normality and poles lying in fundamental domains.

4.3. Theorem. Let $W$ be an additive automorphic function with respect to $\Gamma$, where $\Gamma$ is of the first kind. Then $W$ is a $F_{0}$-normal function in $D$.

Proof. Suppose, on the contrary, that $W$ is not a $F_{0}$-normal function in $D$. By 4.1 there exist a positive real number $R$ and a sequence of points $\left(z_{n}\right) \subset G_{R}$ such that

$$
\lim _{n \rightarrow \infty}\left(1-\left|z_{n}\right|^{2}\right) \frac{\left|W^{\prime}\left(z_{n}\right)\right|}{1+\left|W\left(z_{n}\right)\right|^{2}}=\infty .
$$

Since $W$ is meromorphic in $D$, we have $\left|z_{n}\right| \rightarrow 1$ for $n \rightarrow \infty$. Further, the inequality 
$d\left(z_{n}, F_{0}\right)<R$ implies that at least some subsequence denoted also by $\left(z_{n}\right)$ converges to a parabolic vertex $p$. By the assumption $W$ has an angular limit at $p$. Now $\left(z_{n}\right) \subset \alpha$ where $\alpha$ is a Stolz angle at $p$. Hence

$$
\lim _{n \rightarrow \infty}\left(1-\left|z_{n}\right|^{2}\right) \frac{\left|W^{\prime}\left(z_{n}\right)\right|}{1+\left|W\left(z_{n}\right)\right|^{2}}=0,
$$

which contradicts (4.6). Thus the theorem is proved.

4.4. Remark. Generally speaking we may say that poles do not remove $F_{0}$ normality. This is a difference compared to normality for additive automorphic functions [4, 2.1 Theorem].

4.5. Theorem. Let $W$ be an additive automorphic function with respect to $\Gamma$ where $\Gamma$ is of the second kind. If $\iint_{F_{0}}\left(\left|W^{\prime}(z)\right| /\left(1+|W(z)|^{2}\right)\right)^{2} d \sigma_{z}<\infty$, then $W$ is a $F_{0}$-normal function in $D$.

Proof. Let $\left\{A_{T_{i}}\right\}_{i=1}^{m}$ be the period set of generating transformations $T_{i}$ of $\Gamma$ corresponding to $F_{0}$. By the boundedness of the integral $\iint_{F_{0}}\left(\left|W^{\prime}(z)\right| /\left(1+|W(z)|^{2}\right)\right)^{2} d \varrho_{z}$ we obtain

$$
\iint_{T_{i}^{ \pm \pm} \perp_{\left(F_{0}\right)}}\left(\frac{\left|W^{\prime}(w)\right|}{1+|W(w)|^{2}}\right)^{2} d \sigma_{w}=\iint_{F_{0}}\left(\frac{\left|W^{\prime}(z)\right|}{1+\left|W(z) \pm A_{T_{i}}\right|^{2}}\right)^{2} d \sigma_{z}<\infty
$$

for each $i=1, \ldots, m$. Hence

$$
\iint_{\cup_{i=0}^{m}} \int_{T_{i}^{ \pm 1}\left(F_{0}\right)}\left(\frac{\left|W^{\prime}(w)\right|}{1+|W(w)|^{2}}\right)^{2} d \sigma_{w}=\sum_{i=0}^{m} \iint_{T_{i}^{ \pm 1}\left(F_{0}\right)}\left(\frac{\left|W^{\prime}(w)\right|}{1+|W(w)|^{2}}\right)^{2} d \sigma_{w}<\infty
$$

with $T_{0}=$ id. Assume that the theorem is false. By 4.1 we can find a sequence of points $\left(z_{n}\right) \subset G_{R}$ such that $\left(1-\left|z_{n}\right|^{2}\right)\left|W^{\prime}\left(z_{n}\right)\right| /\left(1+\left|W\left(z_{n}\right)\right|^{2}\right) \rightarrow \infty$ for $n \rightarrow \infty$. Thus $\left(z_{n}\right)$ is a sequence of $P$-points for $W$. From the meromorphic behaviour of $W$ at parabolic vertices it follows that $\left(z_{n}\right)$ tends to a free side. Without loss of generality, it may be supposed $\bigcup_{n=1}^{\infty} U\left(z_{n}, r\right) \subset \bigcup_{i=0}^{m} T_{i}^{ \pm 1}\left(\bar{F}_{0}\right)$ for some $r>0$. Then $W$ assumes every value, except perhaps two, infinitely often in the set $\bigcup_{n=1}^{\infty} U\left(z_{n}, r\right)$. On the other hand, (4.9) means that $W$ maps the set $\bigcup_{i=0}^{m} T_{i}^{ \pm 1}\left(\bar{F}_{0}\right)$ onto a surface of finite spherical area. This is a contradiction, and thus the theorem is proved.

\section{On properties of $F_{0}$-normal functions}

5.1. We make the following remarks: If an additive automorphic function $W$ is $F_{0}$-normal, then it is $T\left(F_{0}\right)$-normal for every $T \in \Gamma$, i.e. for every sequence of points $\left(z_{n}\right) \subset \overline{T\left(F_{0}\right)}$ the family $\left\{g_{n}(\zeta)\right\}=\left\{W\left(\left(\zeta+z_{n}\right) /\left(1+\bar{z}_{n} \zeta\right)\right)\right\}$ forms a normal family in $D$. Further, if $\left(z_{n}\right) \subset G_{R}=\left\{z \mid d\left(z, F_{0}\right)<R\right\}$ is any sequence of points, then $\left\{g_{n}(\zeta)\right\}=\left\{W\left(\left(\zeta+z_{n}\right) /\left(1+\bar{z}_{n} \zeta\right)\right)\right\}$ forms a normal family in $D$ provided $W$ is a $F_{0^{-}}$ 
normal function. Noting the latter remark, we can leave certain theorems in this chapter unproved, since their proof is similar to that of corresponding theorems in the case of normality. By the above remarks we obtain:

5.2. Theorem. Let $W$ be a $F_{0}$-normal but non-normal additive automorphic function with respect to $\Gamma$ and $\left(z_{n}\right)$ a sequence of $P$-points for $W$. Then the sequence $\left(z_{n}\right)$ cannot be contained in any $G_{R}$ and touches infinitely many closures $\bar{F}_{m}$ of fundamental domains $F_{m}=T_{m}\left(F_{0}\right), T_{m} \in \Gamma$.

Next we give a non- $F_{0}$-normality criterion, which connects with [6, Lemma 3]-

5.3. The orem. Let $W$ be an analytic additive automorphic function with respect to $\Gamma$. Suppose that there exist two sequences of points $\left(z_{n}\right),\left(z_{n}^{\prime}\right)$, both in $G_{R}=$ $\left\{z \mid d\left(z, F_{0}\right)<R\right\}$, such that $d\left(z_{n}, z_{n}^{\prime}\right)<M<\infty$ for each $n=1,2, \ldots, \quad$ and $\lim _{n \rightarrow \infty} W\left(z_{n}\right)=0, \lim _{n \rightarrow \infty} W\left(z_{n}^{\prime}\right)=\infty$. Then $W$ is not a $F_{0}$-normal function in $D$.

Proof. The assertion follows from 5.1 and [6, Lemma 3].

5.4. Corollary. Let $W$ be an analytic $F_{0}$-normal additive automorphic function which is not a Bloch function in D. Let $\left(z_{n}\right) \subset F_{0}$ be a sequence of points such that $\left(1-\left|z_{n}\right|^{2}\right)\left|W^{\prime}\left(z_{n}\right)\right| \rightarrow \infty$ for $n \rightarrow \infty$. If $\left(z_{n}^{\prime}\right)$ is any sequence of points such that $d\left(z_{n}, z_{n}^{\prime}\right)<$ $M<\infty$ for each $n=1,2, \ldots$, then $\lim _{n \rightarrow \infty} W\left(z_{n}^{\prime}\right)=\infty$.

Denote by $z_{n}(\alpha), n=1,2, \ldots$, the points where the additive automorphic function $W$ assumes the value $\alpha$. Let $\alpha \neq \beta$.

5.5. Theorem. Let $W$ be a $F_{0}$-normal additive automorphic function with respect to $\Gamma$. If

$$
\inf _{\substack{n=1,2, \ldots \\ m=1,2, \ldots}} d\left(z_{n}(\alpha), z_{m}(\beta)\right)=0
$$

then both the sequence $\left(z_{n}(\alpha)\right)$ and $\left(z_{m}(\beta)\right)$ touch infinitely many closures $\bar{F}_{h}$ of fundamental domains $F_{h}=T_{h}\left(F_{0}\right), T_{h} \in \Gamma$, and neither can lie in any $G_{R}$.

Proof. Suppose first, on the contrary, that there is a subsequence $\left(z_{j}(\alpha)\right) \subset \bar{F}_{h}$ for some $h=0,1,2, \ldots$. Further, we may assume $\lim _{j \rightarrow \infty} d\left(z_{j}(\alpha), z_{j}(\beta)\right)=0$. By 5.1 the function $W$ is $F_{h}$-normal, and as in 4.1 we obtain

$$
\frac{\left|W^{\prime}(z)\right|}{1+|W(z)|^{2}} \leqq \frac{C_{R}}{1-|z|^{2}}
$$

for all $z \in G_{R h}=\left\{z \mid d\left(z, F_{h}\right)<R\right\}$. By integrating it follows

$$
d^{*}\left(W\left(z_{1}\right), W\left(z_{2}\right)\right) \leqq C_{R} d\left(z_{1}, z_{2}\right), \quad z_{1}, z_{2} \in G_{R h} .
$$

Consequently,

$$
\begin{gathered}
0<d^{*}(\alpha, \beta)=d^{*}\left(W\left(z_{j}(\alpha)\right), W\left(z_{j}(\beta)\right)\right) \\
\leqq C_{R} d\left(z_{j}(\alpha), z_{j}(\beta)\right) \rightarrow 0
\end{gathered}
$$

for $j \rightarrow \infty$. This is a contradiction, and thus the first assertion is proved.

Since the similar inequality to in (5.1) holds in any hull $G_{R}$, the latter assertion can be proved exactly as above. 
Next we consider $F_{0}$-normal additive automorphic functions of the first kind.

5.6. Theorem. Let $W$ be an analytic $F_{0}$-normal additive automorphic function of the first kind. Then $W$ is bounded in the fundamental domain $F_{0}$.

Proof. Suppose, one the contrary, that there is a sequence of points $\left(z_{n}\right) \subset F_{0}$ such that $W\left(z_{n}\right) \rightarrow \infty$ for $n \rightarrow \infty$. We form the functions

$$
g_{n}(\zeta)=W\left(\frac{\zeta+z_{n}}{1+\bar{z}_{n} \zeta}\right), \quad n=1,2, \ldots
$$

By the assumption $\left\{g_{n}\right\}$ is a normal family in $D$. Hence there is a subsequence $\left(g_{k}\right)$ of $\left(g_{n}\right)$ such that $\lim _{k \rightarrow \infty} g_{k}(\zeta)=g(\zeta)$ uniformly on every compact part of $D$. Further, $g$ is an analytic function in $D$. Therefore

$$
\lim _{k \rightarrow \infty} W\left(z_{k}\right)=\lim _{k \rightarrow \infty} g_{k}(0)=g(0) \neq \infty,
$$

which is a contradiction. Thus the proof is complete.

5.7. Remark. If $W$ is an analytic $F_{0}$-normal additive automorphic function of the first kind, then $W$ is a Bloch function by [11, Theorem 3].

Compared to 2.2 we obtain:

5.8. Theorem. Let $W$ be a $F_{0}$-normal additive automorphic function with respect to $\Gamma$. Then $W$ is of the first kind if and only if

$$
\liminf _{a \in G_{R}} \iint_{U(a, r)}\left(\frac{\left|W^{\prime}(z)\right|}{1+|W(z)|^{2}}\right)^{2} d \sigma_{z}>0
$$

for any positive number $r$ and any hull $G_{R}=\left\{z \mid d\left(z, F_{0}\right)<R\right\}$.

Proof. The assertion follows from 2.2 and 5.1.

5.9. Theorem. Let $W$ be an analytic $F_{0}$-normal additive automorphic function of the first kind and $r$ any positive number. Then there exists a positive number $m_{r}$ such that

$$
d^{*}(W(z), \alpha)>m_{r} \quad \text { in } \quad D-\bigcup_{n=1}^{\infty} U\left(z_{n}(\alpha), r\right),
$$

where $z_{n}(\alpha), n=1,2, \ldots$, denote the $\alpha$-points of $W$.

Proof. (Cf. [13, Theorem 1 (iii)].) Suppose, on the contrary, that there exists a positive number $r$ such that

$$
\inf _{z \in D-\cup_{n=1}^{\infty} U\left(z_{n}(\alpha), r\right)} d^{*}(W(z), \alpha)=0 .
$$

Then there exists a sequence of points $\left(z_{k}\right)$ in $D-\cup_{n=1}^{\infty} U\left(z_{n}(\alpha), r\right)$ such that $\lim _{k \rightarrow \infty} W\left(z_{k}\right)=\alpha$. Let $z_{k}^{\prime}=T_{k}\left(z_{k}\right) \in \bar{F}_{0}$ and put $g_{k}(\zeta)=W\left(\left(\zeta+z_{k}^{\prime}\right) /\left(1+\bar{z}_{k}^{\prime} \zeta\right)\right)$. By the assumption there is a subsequence denoted also by $\left(g_{k}\right)$ converging uniformly to 
a non-constant analytic limiting function $g$ on each compact subset of $D$. Then

$$
\begin{aligned}
g(0) & =\lim _{k \rightarrow \infty} g_{k}(0)=\lim _{k \rightarrow \infty} W\left(z_{k}^{\prime}\right)=\lim _{k \rightarrow \infty} W\left(T_{k}\left(z_{k}\right)\right) \\
= & \lim _{k \rightarrow \infty}\left(W\left(z_{k}\right)+A_{T_{k}}\right)=\alpha+\lim _{k \rightarrow \infty} A_{T_{k}} \neq \infty .
\end{aligned}
$$

Denote $\quad S_{k}(\zeta)=\left(\zeta+z_{k}^{\prime}\right) /\left(1+\bar{z}_{k}^{\prime} \zeta\right) \quad$ and $\quad h_{k}(\zeta)=W\left(T_{k}^{-1}\left(S_{k}(\zeta)\right)\right)=W\left(S_{k}(\zeta)\right)-A_{T_{k}}=$ $g_{k}(\zeta)-A_{T_{k}}$. Hence the sequence of functions $\left(h_{k}\right)$ converges uniformly to a nonconstant analytic function $h=g-\lim _{k \rightarrow \infty} A_{T_{k}}$ on every compact part of $D$ and $h(0)=\alpha$. By the Hurwitz theorem we may choose a sequence of points $\left(\zeta_{k}\right) \subset$ $U\left(0, r_{1}\right), \quad 0<r_{1}<r / 2, \quad$ such that $h_{k}\left(\zeta_{k}\right)=\alpha$. Consequently, $\quad \alpha=g_{k}\left(\zeta_{k}\right)-A_{T_{k}}=$ $W\left(S_{k}\left(\zeta_{k}\right)\right)-A_{T_{k}}=W\left(T_{k}^{-1}\left(S_{k}\left(\zeta_{k}\right)\right)\right)=W\left(w_{k}\right)$, where $w_{k}=T_{k}^{-1}\left(S_{k}\left(\zeta_{k}\right)\right) \in U\left(z_{k}, r_{1}\right)$. Hence $w_{k} \notin U\left(z_{n}(\alpha), r_{1}\right)$ for all $k$ and all $n$. This is a contradiction, and thus the theorem is proved.

5.10. Theorem. Let $W$ be an analytic $F_{0}$-normal additive automorphic function of the first kind and $\alpha, \beta$ any two values. Then

$$
\inf _{\substack{v=1,2, \ldots \\ \mu=1,2, \ldots}} d\left(z_{v}(\alpha), z_{\mu}(\beta)\right)>0
$$

where $z_{v}(\alpha)$ and $z_{\mu}(\beta)$ denote the $\alpha$-points and $\beta$-points of $W$, respectively.

Proof. Suppose, on the contrary, that there are two values $\alpha$ and $\beta(\alpha \neq \beta)$ such that

$$
\inf _{\substack{v=1,2, \ldots \\ \mu=1,2, \ldots}} d\left(z_{v}(\alpha), z_{\mu}(\beta)\right)=0 .
$$

Then there are subsequences $\left(z_{n}^{\prime}\right)$ and $\left(z_{n}^{\prime \prime}\right)$ of $\left(z_{v}(\alpha)\right)$ and $\left(z_{\mu}(\beta)\right)$ such that

$$
\lim _{n \rightarrow \infty} d\left(z_{n}^{\prime}, z_{n}^{\prime \prime}\right)=0 \text {. }
$$

Let $T_{n} \in \Gamma$ be such that $T_{n}\left(z_{n}^{\prime}\right) \in \bar{F}_{0}$. We form the functions $g_{n}(\zeta)=W\left(\left(\zeta+T_{n}\left(z_{n}^{\prime}\right)\right)\right.$ ! $\left.\left(1+\overline{T_{n}\left(z_{n}^{\prime}\right)} \zeta\right)\right)$. By the assumption one can find a subsequence $\left(g_{k}\right)$ converging uniformly to an analytic function $g$ on every compact part of $D$. Let $\zeta_{k}=$ $\left(T_{k}\left(z_{k}^{\prime \prime}\right)-T_{k}\left(z_{k}^{\prime}\right)\right) /\left(1-\overline{T_{k}\left(z_{k}^{\prime}\right)} T_{k}\left(z_{k}^{\prime \prime}\right)\right) . \quad$ Now $\quad d\left(0, \zeta_{k}\right)=d\left(T_{k}\left(z_{k}^{\prime}\right), T_{k}\left(z_{k}^{\prime \prime}\right)\right)=d\left(z_{k}^{\prime}, z_{k}^{\prime \prime}\right) \rightarrow 0$ for $k \rightarrow \infty$. Thus

$$
\begin{gathered}
\lim _{k \rightarrow \infty} g_{k}(0)=\lim _{k \rightarrow \infty} W\left(T_{k}\left(z_{k}^{\prime}\right)\right)=\lim _{k \rightarrow \infty}\left(W\left(z_{k}^{\prime}\right)+A_{T_{k}}\right) \\
=\alpha+\lim _{k \rightarrow \infty} A_{T_{k}}=\lim _{k \rightarrow \infty} g_{k}\left(\zeta_{k}\right)=\lim _{k \rightarrow \infty} W\left(T_{k}\left(z_{k}^{\prime \prime}\right)\right) \\
=\lim _{k \rightarrow \infty}\left(W\left(z_{k}^{\prime \prime}\right)+A_{T_{k}}\right)=\beta+\lim _{k \rightarrow \infty} A_{T_{k}} .
\end{gathered}
$$

But this is a contradiction, since

The theorem follows.

$$
\lim _{k \rightarrow \infty} A_{T_{k}}=g(0)-\alpha \neq \infty .
$$

5.11. Remark. The fact that $W$ is of the first kind is essential unlike in [13, Theorem 1 (ii)]. 
5.12. Theorem. Let $W$ be a $F_{0}$-normal additive automorphic function with respect to $\Gamma$, and let $\gamma$ be a path in $G_{R}$ whose end is a point $z_{0} \in \partial D$. If $W$ has the limit value $c$ along $\gamma$, then $W$ has the angular limit $c$ at $z_{0}$.

Proof. The theorem follows from 5.1 and [8, Theorem 2].

5.13. We close the chapter by giving a sufficient condition for a $F_{0}$-normal additive automorphic function to be normal in $D$.

Let $W$ be an analytic $F_{0}$-normal additive automorphic function in $D$. If we do not allow the normal family $\left\{g_{n}(\zeta)\right\}=\left\{W\left(\left(\zeta+z_{n}\right) /\left(1+\bar{z}_{n} \zeta\right)\right)\right\}$ to have the constant $\infty$ as a limiting function for any sequence of points $\left(z_{n}\right) \subset \bar{F}_{0}$, then $W$ is a normal function in $D$. In fact, we can prove $W$ to be a Bloch function in $D$ in the same way as in 5.6.

\section{References}

[1] Aulaskari, R.: Additive automorphic functions of the first kind. - Publ. of the Univ. of Joensuu Ser. B 16, 1979, 1-12.

[2] Aulaskari, R.: On additive automorphic functions of the first kind. - Reports of the Department of Mathematics, University of Helsinki, Ser. A No. 18, 1978, 1-11.

[3] Aulaskari, R.: On $F_{0}$-normal additive automorphic functions. - Reports of the Department of Mathematics, University of Helsinki, Ser. A No. 22, 1979, 1-15.

[4] Aulaskari, R.: On normal additive automorphic functions. - Ann. Acad. Sci. Fenn. Ser. A I Math. Dissertationes 23, 1978, 1-53.

[5] Hille, E.: Analytic function theory, Volume II. - Blaisdell Publishing Company, Waltham, Massachusetts-Toronto-London, 1962.

[6] LAppan, P.: Non-normal sums and products of unbounded normal functions. - Michigan Math. J. 8, 1961, 187-192.

[7] LeHNER, J.: Discontinuous groups and automorphic functions. - American Mathematical Society, Providence, Rhode Island, 1964.

[8] Lehto, O., and K. I. Virtanen: Boundary behaviour and normal meromorphic functions. Acta Math. 97, 1957, 47-65.

[9] Niebur, D., and M. Sheingorn: Characterization of Fuchsian groups whose integrable forms are bounded. - Ann. of Math. 106, 1977, 239-258.

[10] Noshiro, K.: Contributions to the theory of meromorphic functions in the unit-circle. - J. Fac. Sci. Hokkaido Imp. Univ. Ser. I 7, 1938, 149-157.

[11] Pommerenke, $\mathrm{CH}_{\text {. }}$ : On inclusion relations for spaces of automorphic forms. - Lecture Notes in Mathematics 505, Springer-Verlag, Berlin-Heidelberg-New York, 1976.

[12] TsE, K.: Some results on value distribution of meromorphic functions in the unit disk. - Nagoya Math. J. 34, 1969, 105-119.

[13] ZINNO, T.: On some properties of normal meromorphic functions in the unit disc. - Nagoya Math. J. 33, 1968, 153-164.

University of Joensuu

Department of Mathematics and Physics

SF-80100 Joensuu 10

Finland

Received 12 October 1979 